\title{
Coping Intervention and Degree of Depression Observed among the Mothers of Mentally Challenged Children
}

\author{
Shylaja Krupanidhi ${ }^{1}$, Dr. Rebecca Samson ${ }^{2}$ \\ ${ }^{1}$ (Phd Student at the Time of Study) \\ ${ }^{2}$ Dean, PIMS College of Nursing Pondicherry.
}

\begin{abstract}
The present study was conducted to explore the degree of depression observed among the mothers of Mentally Challenged Children and to evaluate the effectiveness of structured coping intervention on the degree of depression. The mothers, who have mentally challenged children and have enrolled these children in Institutions for Special Children in Mysore, were considered for the research investigation. The research design selected for the present study was Experimental Before Intervention and After Intervention with Control Group. A total of 120 mothers, who fulfilled the inclusion and exclusion criteria were considered for the study following the pilot study. There were 60 mothers in experimental and 60 mothers in control group. Data was collected by using semi structured interview schedule. The degree of depression was assessed by a semi structured interview schedule that focused on the assessment of depression among the mothers of mentally challenged children. It comprised of 60 items under 10 sub scales. A Structured Coping Intervention for experimental group was administered consisting of Psycho-education, Planning and Implementation of Daily Activity Schedule along with choosing a Fun Activity and performing Progressive Muscle Relaxation Exercise. Structured coping intervention was administered for 45 minutes and advised to practice for seven days. Group consisted of 4-5 members per session. PPT assisted psycho education was given. Progressive Muscle Relaxation Exercise was demonstrated and requested the mothers of experimental group to re do the same. The experimental group respondents were issued Booklets on coping interventions as reference to practice at home for a period of 7 days. The tool was re-distributed and data was collected the data was Collected for further analysis. In the experimental group, Out of 60 mothers, 20\% had mild depression, 61.7\% had moderate depression and 18.3\% had severe depression before intervention. After the coping intervention the results revealed that $100 \%$ of the mothers, with mentally challenged children, had only mild depression. In the control group, out of 60 mothers of mentally challenged children 3.4\% had mild depression, $48.3 \%$ had moderate depression and $48.3 \%$ had severe depression. After intervention (without intervention) the result showed that majority of mothers 'i.e $86.74 \%$ had severe depression and $13.3 \%$ had moderate depression. There was an overall significant relationship between the study groups and degree of depression found at $P<0.001$ before and after intervention. The findings of the present study have implication in understanding that mothers of mentally challenged children do suffer with different degree of depression and structured coping intervention has an effect in reducing the degree of depression. The coping intervention was found to be effective in reducing the Degree of Depression among the Mothers of Mentally Challenged Children.
\end{abstract}

\section{Introduction}

Motherhood is the gift of God. Every mother hopes for a healthy normal child. She lives with hope for nine months to have a healthy baby. It is an agony, pain and depression when she comes to know that her child is mentally challenged. It is more painful for her when she understands that the child has to live with this problem which is life time. The birth of a mentally challenged child in to a family may give rise to negative feelings which can be found in parents of disabled children at early and later periods in the life-cycle and they are the following: protectiveness of the helpless; revulsion at the abnormal; inadequacy of reproduction; inadequacy of rearing; anger; grief; shock; guilt; embarrassment (Mac Keith (1973) and Dale (1996). Parents of mental challenged children often experience feelings of stress and anxiety (Holroyd, Brown, Wikler and Simmons 1975). Carpiniello, Piros, Pariente and Carta (1995) also revealed that parents of disabled children had significantly higher levels of psychiatric symptoms and were more likely to meet criteria for depressive disorders, compared with controls. Between the parents of mentally challenged children and neurologically impaired children there was no significant difference. Hannam (1975) reported that the presence of mentally retarded child alters the normal chores of the family. Holroyd and Guthrie (1979) found that mothers generally feel burdened and the family members deprived of normal family life because of the presence of a handicapped.

Though a disabled child is problematic in every society and culture, the psychological reaction to this, to a large extent, affects the family members specially the mother who is the care taker of the children especially in our society and culture and it is the felt need that the parents need a coping intervention to combat early with the present problem. 


\section{Aims of the Study}

1. To prepare a structured coping intervention module for the mothers of mentally challenged children.

2. To impart the coping intervention to the mothers of mentally challenged children

\section{Objectives of the Study}

1. To assess the degree of depression among the mothers of mentally challenged children before implementation of coping intervention.

2. To assess the degree of depression for both control and experimental group after implementation of coping intervention.

3. To develop and validate the booklet of coping intervention for depressed mothers with mentally challenged children.

4. To assess the effectiveness of coping intervention for depression on the mothers with mentally challenged children

5. To associate the selected socio-demographic variables with the degree of depression among the mothers of mentally challenged children.

\section{Methodology}

Research Approach The research approach adopted for the present study was an Evaluative Approach. This approach was adopted to evaluate the effectiveness of structured coping intervention on the degree of depression observed among the mothers of mentally challenged children

Research Design The research design selected for the present study was Experimental Before Intervention and After Intervention with Control Group. In this study psycho-education, planning and implementation of daily activity schedule along with choosing a fun activity and performing progressive muscle relaxation exercise were used as the structured coping intervention.

Setting of the Study: The setting for the present study was, selected institutions for special children at Mysore. Out of 16 institutions for special children, 4 institutions for special children were selected for the study randomly. Out of these, two institutions were chosen for experimental, and the other 2 for control group at random.

Sample: Sample for the present study, was Mothers of Mentally Challenged Children whose children are enrolled in institutions for special children and the Mothers who bring their Mentally Challenged Children for speech therapy and physiotherapy on OPD basis.

Sample Size: The sample size comprised of 120 respondents, 60 in experimental group and 60 in the control group.

Sampling Technique: Multi staged sampling technique was followed to select the institution for special children.

After selecting the institution for experimental and control group the respondents were selected by random sampling technique preparing a sample frame.

\section{Description of Research Tool}

For the present study, the investigator constructed a semi structured interview schedule consisting of 2 sections i.e. section A and section B.

Section A. Deals with Socio-demographic sheet consisting of two parts.

Part I Is a semi structured interview schedule focusing on the background information of the child, consisting of 6 questions regarding degree of mental retardation of the child, any associated conditions, age, gender, birth order of the child and no. of children.

Part II Focuses on the particulars of the respondents having 11 questions regarding age of the mother, religion, education, occupation, family income per month, marital status, habitat, consanguinity of marriage, family type, total no. of people staying in the house, including the mother and the child and family history of having a mentally challenged child.

Section B Is a semi structured interview schedule consisting of 10 subscales focusing on the assessment of different aspects of depression for mothers of mentally challenged children. 
Table 1 Depression Assessment Scale for Mothers of Mentally Challenged Children, consisting of 10 sub-

scales.

\begin{tabular}{|l|l|l|l|}
\hline Sl. No & Sub scale & Area of assessment depression & No. of items \\
\hline 01 & 1 & Personal distress & 15 \\
\hline 02 & 2 & Daily care & 06 \\
\hline 03 & 3 & Neglect of others & 03 \\
\hline 04 & 4 & Disturbed behavior & 03 \\
\hline 05 & 5 & Marital problems & 07 \\
\hline 06 & 6 & Other interpersonal problems & 02 \\
\hline 07 & 7 & Effect of sisblings and other family members & 06 \\
\hline 08 & 8 & Altered social life & 06 \\
\hline 09 & 9 & Social embarrassment & 06 \\
\hline 10 & 10 & $\begin{array}{l}\text { Financial implication } \\
\text { TOTAL }\end{array}$ & 06 \\
& & $\mathbf{6 0}$ \\
\hline
\end{tabular}

Content Validity: -. The experts in the specialty area were requested to give their suggestion towards the clarity, simplicity and meaningfulness..

Reliability:- The reliability of the tool was established using split half method which measures the coefficient of internal consistency. The reliability was calculated by using Karl Pearson correlation coefficient formula developed by Brown Prophecy. The reliability of the test was found to be $r=0.841(>0.7)$ for the depression scale. Hence the tool was found to be statistically reliable for the study.

Translation of the tool: The finalized tool was translated into Kannada. To determine the correctness it was retranslated to English and then back to Kannada.

Procedure for Data Collection

The data was collected after getting formal written consent from the authorities and the subjects prior to the study.

\section{Phases of Data Collection}

\section{Phase I: Before intervention, coping intervention and distribution of booklet}

The tool to assess the degree of depression for the Mothers of Mentally Challenged Children was administered to both the groups after giving a detailed explanation and getting their consent. The subjects were instructed to respond to all the items as there was no right or wrong answers.

After completion of the tool the respondents of experimental group ( $\mathrm{N}=60)$ Were administered with coping intervention that included Psycho-education, Planning and Implementation of Daily Activity Schedule along with choosing a Fun Activity and performing Progressive Muscle Relaxation Exercise for 45 minutes. PPT was used for psycho education and for Progressive Muscle Relaxation Exercise; demonstration with return demonstration method was adopted. The experimental group respondents were also issued a booklet for their reference to practice the coping intervention, for a period of 7 days.

\section{Phase II: After Intervention}

In this phase the subjects $(\mathrm{N}=120)$ were called after seven days they were all assembled at the same venue at a particular time. The same tool was re distributed and data was collected. The collected data was analyzed.

\section{Salient Findings}

1. Out of 120 children, majority of the children 55(45.8\%) had moderate mental retardation. Majority of the children $27(22.5 \%$ ) had fits along with mental retardation, 52(43.3\%) were in the age group 1-10 years, On analysis, it was found that male and female ratio was 3:1. Majority of the children i.e. $48.3(58 \%)$ were first born, $63(52.5 \%)$ of the mothers had two children

2. Among 120 mothers of mentally challenged children, majority of the mothers $47(39.2 \%)$ belong to the age group of $31-40,109(90.8 \%)$ were Hindus, 63(52.5\%) had education up to secondary schooling. On analysis it was found that $90(75 \%)$ were housewives. Looking at the family income it was analyzed that $74(61.7 \%)$ samples family income was between Rs. 4500/- and 8000/-

3. Out of 60 mothers in the experimental group, 12(20\%) had mild depression, 37(61.7\%) had moderate depression and 11(18.3\%) had severe depression. After the coping intervention, the results revealed that $60(100 \%)$ of the mothers with mentally challenged children had only mild depression.

4. Out of 60 mothers of mentally challenged children of control group 2(3.4\%) showed mild depression, $29(48.3 \%)$ had moderate depression and 29(48.3\%) were with severe depression. After intervention (without intervention) Majority of mothers i.e., 52(86.74\%) had severe depression and 8(13.3\%) had moderate depression, no mothers with mild depression. A significant relationship was found at found at $\mathrm{P}$ $<.001$ before and after intervention. 
5. On 10 Sub-Scales of Depression Assessment scale for Mothers of Mentally Challenged Children for experimental group $n=60$ and control group $n=60$, it was found highly significant at $P<0.001$ before and after intervention for all the 10 sub-scales.

6. The summarized mean and SD values of experimental and control group, before and after coping intervention was found that the mean \pm SD of experimental group was $1.51 \pm 31.22$ before the intervention. In control group the mean \pm SD was $1.74 \pm 20.21$ before intervention. The mean difference was -23.01 the $t$ value was -4.79 at df 118 which is significant at $\mathrm{p}<.001$.

7. It was identified that after coping intervention the mean \pm SD of experimental group was $77.63 \pm 7.61$ and the mean \pm SD of control group was $2.01 \pm 16.93$. The mean difference was -123.72 the $t$ value was -51.633 , which is significant at $\mathrm{p}<.001$.

8. The experimental group showed no significant association between the selected socio-demographic variables of the mentally challenged children and degree of depression among the mothers, in before intervention and no statistics could be computed as after intervention, the results are constant.

9. There was no significant relationship found with the selected socio demographic variables of the mentally challenged children and the degree of depression among the mothers of control group n-60 (before intervention).

10. There was a significant association found between associated condition of the children and mothers depression at $\mathrm{P}<0.05$ and between age group of child and mothers depression at $\mathrm{P}<0.001$ after intervention (No intervention was given) in the control group.

11. Experimental group n-60 showed that mothers' age, and degree of depression among the mothers was significant, at $\mathrm{p}<.005$ before intervention. No statistics are computed as after intervention, the results are constant.

12. Mothers of control group n 60 showed that, mother's education, history of consanguinity and degree of depression among the mothers was significant at $\mathrm{p}<.05$ and $\mathrm{p}<0.01$ respectively before intervention.

13. Mothers of control group n-60 after intervention showed that mothers' age, education, history of consanguinity of marriage and family type were significant at $\mathrm{p}<0.05, \mathrm{p}<0.01, \mathrm{p}<0.05$ and $\mathrm{p}<0.01$ respectively with degree of depression .

This indicates that mothers of mentally challenged children do suffer with different degree of depression and coping intervention has an effect in reducing the degree of depression.

\section{References}

[1] Araya R et al., (2006); "Cost effectiveness of A Primary Care Treatment Program for Depression in Low-Income Women in Santiago, Chile". Am J Psychiatry, 163:1379-87.

[2] Fartheen A, et al., (2003) "coping strategies in families with mentally retarded children", Indian Journal for the Practicing Doctor. Vol. 30; P 98-104.

[3] Hashim H A et al, (2011) The Effects of Progressive Muscle Relaxation and Autogenic Relaxation on Young Soccer Players' Mood States. Asian J Sports Medicine Jun; 2(2); PP 99-105.

[4] Ram S et al.,(2013)"Effectiveness of Group Psycho-Education on Well Being and Depression Among Breast Cancer Survivors of Melaka, Malaysia", Indian J palliate Care, Jan;19(1):PP34-9.

[5] Sreedevi T R, sukumar P S (2010); "Facilitating Factors of Coping in Mothers of Children with Mental Retardation"; The Nursing Journal of India: October Vol. Cl No. 10

[6] Yildirim A et al,.(2013)effect of Nursing Intervention Program on the Depression and Perseption of Family Functioning of Mothers with Intellectually Disabled Children. J Clin Nurse Jan; 22(1-2):251-61 